Argonne

ANL-21/15

\title{
Design of Microwave Resonant Cavity Transducer
}

Development of Sensor Performance Model of Microwave Cavity Flow Meter for Advanced Reactor High Temperature Fluids

Nuclear Science and Engineering Division 


\title{
About Argonne National Laboratory
}

Argonne is a U.S. Department of Energy laboratory managed by UChicago Argonne, LLC under contract DE-AC02-06CH11357. The Laboratory's main facility is outside Chicago, at 9700 South Cass Avenue, Argonne, Illinois 60439. For information about Argonne and its pioneering science and technology programs, see www.anl.gov.

\section{Document availability}

Online Access: U.S. Department of Energy (DOE) reports produced after 1991 and a growing number of pre-1991 documents are available free at OSTI.GOV

(http://www.osti.gov/), a service of the U.S. Dept. of Energy's Office of Scientific and

Technical Information

\author{
Reports not in digital format may be purchased by the public from the \\ National Technical Information Service (NTIS): \\ U.S. Department of Commerce \\ National Technical Information Service \\ 5301 Shawnee Rd \\ Alexandria, VA 22312 \\ www.ntis.gov \\ Phone: (800) 553-NTIS (6847) or (703) 605-6000 \\ Fax: (703) 605-6900 \\ Email: orders@ntis.gov
}

\author{
Reports not in digital format are available to DOE and DOE contractors from the \\ Office of Scientific and Technical Information (OSTI): \\ U.S. Department of Energy \\ Office of Scientific and Technical Information \\ P.O. Box 62 \\ Oak Ridge, TN 37831-0062 \\ www.osti.gov \\ Phone: (865) 576-8401 \\ Fax: (865) 576-5728 \\ Email: reports@osti.gov
}

Disclaimer

This report was prepared as an account of work sponsored by an agency of the United States Government. Neither the United States Government nor any agency thereof, nor UChicago Argonne, LLC, nor any of their employees or officers, makes any warranty, express or implied, or assumes any legal liability or responsibility for the accuracy, completeness, or usefulness of any information, apparatus, product, or process disclosed, or represents that its use would not infringe privately owned rights. Reference herein to any specific commercial product, process, or service by trade name, trademark, manufacturer, or otherwise, does not necessarily constitute or imply its endorsement, recommendation, or favoring by the United States Government or any agency thereof. The views and opinions of document authors expressed herein do not necessarily state or reflect those of the United States Government or any agency thereof, Argonne National Laboratory, or UChicago Argonne, LLC. 


\section{Design of Microwave Resonant Cavity Transducer}

Development of Sensor Performance Model of Microwave Cavity Flow Meter for Advanced Reactor High Temperature Fluids

prepared by

Alexander Heifetz, Dmitry Shribak, Sasan Bakhtiari, Eugene R. Koehl

Nuclear Science Engineering Division, Argonne National Laboratory

March 31, 2021 


\section{Table of Contents}

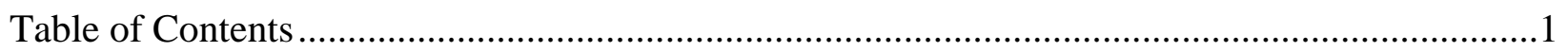

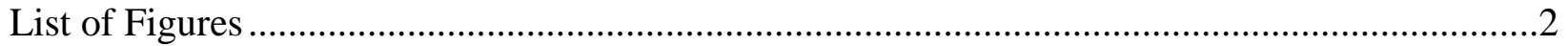

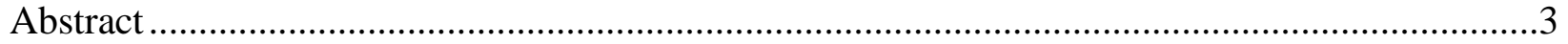

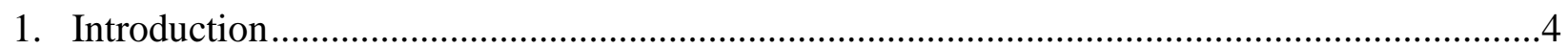

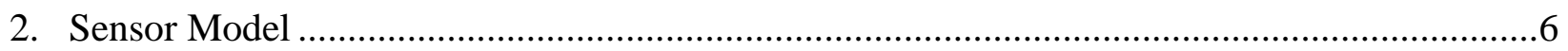

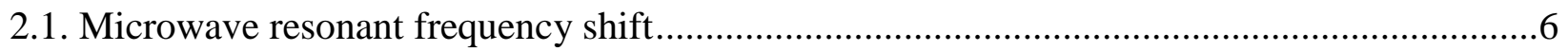

2.2. Thermophysical material properties ...........................................................................

3. Computer Simulations of Sensor Performance ..............................................................

3.1. Selection of transducer geometric parameters ..............................................................9

3.2. Computer simulations of microwave resonant frequency shift .......................................10

3.3. Coupling of microwave field into cylindrical resonator ..................................................14

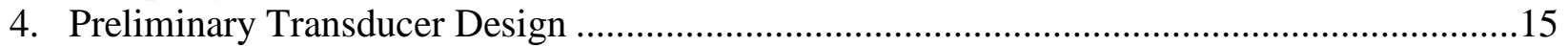

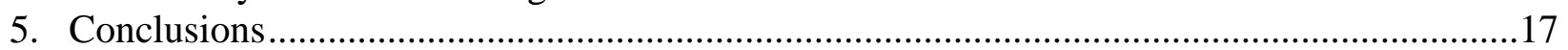

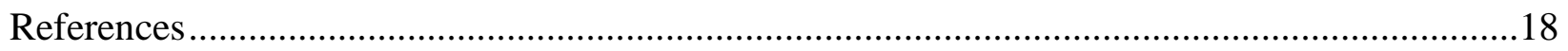




\section{List of Figures}

Figure 1 - (a) Conceptual drawing of waveguide-coupled microwave cylindrical cavity with flexible membrane. (b) Deflection of membrane due to dynamic fluid pressure changes cavity volume, which shifts microwave resonant frequency.

Figure 2 - Visualization of $\mathrm{TM}_{011}$ mode in cylindrical cavity with $R=0.8 \mathrm{in}$ occuring at $15.4 \mathrm{GHz}$. Flexible membrane with $d=10 \mathrm{mil}$ is at the top of the cylinder.... 10

Figure 3 - (a) Conceptual Figure 3. Frequency shift of $\mathrm{TM}_{011}$ mode resonant frequency of $R=0.4 \mathrm{in}$ and $d=10$ mil stainelss steel cylinder due to liquid sodium flow-induced membrane deflections at $500^{\circ} \mathrm{C}$. Frequency shifts are calculated with COMSOL RF module using curved membrane model Equation (2), with COMSOL RF using flat membrane model with equivalent amplitude $w_{\text {avg }}=$ $w_{0} / 3$, and analytically using Equation (10). 11 Figure 4 - Visualization of $\mathrm{TE}_{011}$ mode in right cylindrical cavity with $R=0.4$ in occuring at $f_{0}=$ $22.2 \mathrm{GHz}$. Flexible membrane with $d=10 \mathrm{mil}$ is at the top of the cylinder 12 Figure 5 - Frequency shift of $\mathrm{TE}_{011}$ mode resonant frequency shift of $R=0.4 \mathrm{in}$ and $d=10 \mathrm{mil}$ stainless steel cylinder due to liquid sodium flow-induced membrane deflections at $500^{\circ} \mathrm{C}$. Frequency shift is calculated with COMSOL RF module using curved membrane model......... 12 Figure 6 - Visualization of $\mathrm{TE}_{012}$ mode in right cylindrical cavity with $R=0.4$ in occuring at $f_{0}=$ $26.5 \mathrm{GHz}$. Flexible membrane with $d=10 \mathrm{mil}$ is at the top of the cylinder 13

Figure 7 - Frequency shift of $\mathrm{TE}_{012}$ mode resonant frequency shift of $R=0.4$ in and $d=10 \mathrm{mil}$ stainless steel cylinder due to liquid sodium flow-induced membrane deflections at $500^{\circ} \mathrm{C}$. Frequency shift is calculated with COMSOL RF module using curved membrane model.......... 13 Figure 8 - Dependence of $\mathrm{Q}$ factor of $\mathrm{TE}_{012}$ mode on the radius of a circular aperture in the side wall of the cylindrical cavity 14

Figure 9 - Side view and 3D visualization of the cylindrical cavity design. 15 Figure 10 - Top view of the cavity and 3D visualization of waveguide coupling cavity side wall 


\section{Abstract}

High-temperature fluid reactors, such as molten salt cooled reactors (MSCR) and sodium fast reactors (SFR), are a promising advanced reactor option. Measurement of high-temperature fluid process variables, in particular the flow inside the pressure vessel, is a challenging task because of harsh environment, which includes high radiation, high temperature, and contact with highly corrosive coolant fluid. We are investigating a microwave cavity-based transducer for hightemperature fluid flow sensing. This sensor is a hollow metallic cylindrical cavity, which can be fabricated from stainless steel, and as such is expected to be resilient to radiation, high temperature and corrosive environment of MSCR and SFR. The principle of sensing consists of making one wall of the cylindrical cavity flexible enough so that dynamic pressure, which is proportional to fluid velocity, will cause membrane deflection. A cavity is characterized by its resonant frequencies. Membrane deflection causes cavity volume change, which leads to a shift in the resonant frequency. Feasibility of the flow sensor is evaluated with signal sensitivity using COMSOL computer simulations. A right cylinder geometry stainless steel cavity with dimeter of 0.8 in was investigated. We choose membrane thickness of $10 \mathrm{mil}$, so that corrosion anticipated to proceed at the rate of 1 mil/year in liquid sodium would affect no more than $10 \%$ of the membrane. Using the properties of liquid sodium fluid, and stainless-steel material property values at $500^{\circ} \mathrm{C}$, we calculate frequency shift for a range of values of fluid velocity from $0.5 \mathrm{~m} / \mathrm{s}$ to $2 \mathrm{~m} / \mathrm{s}$. Results of computer simulations indicate measurable sensitivity to flow for this cavity design. Following these simulations, we have developed a preliminary design for fabrication of a transducer operating in microwave K-band for proof-of-principle tests. 


\section{Introduction}

High-temperature fluid reactors, such as sodium fast reactors (SFR) and molten salt cooled reactors (MSCR) are a promising advanced reactor option with highly efficient thermal energy conversion cycle [1-3]. Streamlining commercialization of advanced reactors involves development of new coolant sensing technologies for enhancing performance efficiency [4,5]. Measurement of high-temperature fluid process variables, in particular the flow inside the pressure vessel, is a challenging task because of harsh environments of advanced reactors, which includes radiation, high temperature, and contact with highly corrosive coolant fluid. Liquid metal flow sensors are typically of magnetic, Venturi and ultrasonic types [4,5], while molten salt flow sensors are ultrasonic [6]. Magnetic flow meters, which consist of high temperature permanent magnets (PM) and flux measurement coil (saddle coil), measure the rate of conducting flux passing through coil cross-section. The challenges of PM include heavy weight, temperature dependent output, and drifting with time. Difficulties with coil-based flow meters include large size of the DC power supply, temperature dependent output, nonlinearity at larger scales, and dependence on flow profile. Challenges with Venturi flow meters include long installation length, dependence on flow profile, and poor precision at low flow velocity. Ultrasonic flow meters are based on detecting either the time of flight or the Doppler frequency shift of acoustic waves in pitch-and-catch or in transmission mode. Ultrasonic transducers can be placed outside the pressure vessel. However, ultrasonic sensing requires a direct line-of-sight between the transducers. In addition, ultrasonic flowmeters are sensitive to flow profile in single path configuration, and thus might involve a multipath arrangement and thus complicated signal processing.

We are investigating a microwave cavity-based transducer for high-temperature fluid flow sensing. This sensor is a hollow metallic cavity, which can be fabricated from stainless steel, and as such is expected to be resilient to radiation, high temperature and corrosive environment of SFR and MSCR. A viable geometry of the sensor is that of a small cylindrical resonator [7,8]. A schematic drawing of the flow sensor is shown in Figure 1. The principle of sensing consists of making one wall of the cavity flexible enough so that dynamic pressure, which is proportional to fluid velocity, will cause membrane deflection. A cavity is characterized by its resonant frequencies, which occur due to constructive interferences of microwave field inside the cavity. Membrane deflection causes cavity volume change, and thus a shift in the resonant frequency. Using signal readout from microwave frequency shift in a hollow cavity is advantageous for applications in high temperature and high radiation environment, because no electronic components are placed inside the transducer. Since the microwave cavity sensing is based on fluidstructure interaction, as opposed to fluid electrical conductivity, the proposed sensor is equally applicable to liquid sodium and molten salt flow sensing. Energy coupling to and from the sensor will be achieved through a microwave waveguide, which will be an integral part of the insertion probe. A waveguide is a rigid narrow hollow metallic tube resilient to high temperature and highradiation environment. In the drawing in Figure 1, microwave field is coupled into the cylindrical resonant cavity through a side wall. This is typically accomplished by making a subwavelengthsize hole on the side of the cavity. 


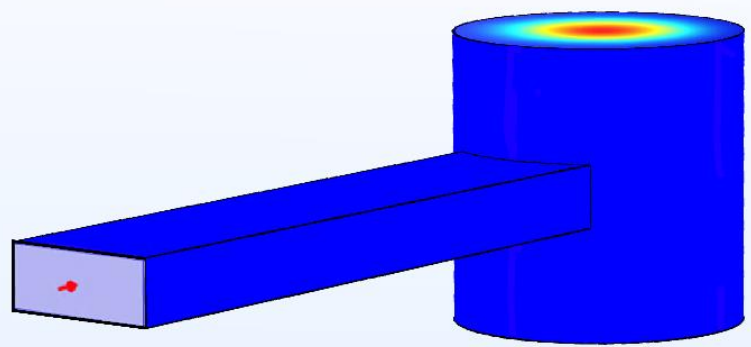

(a)

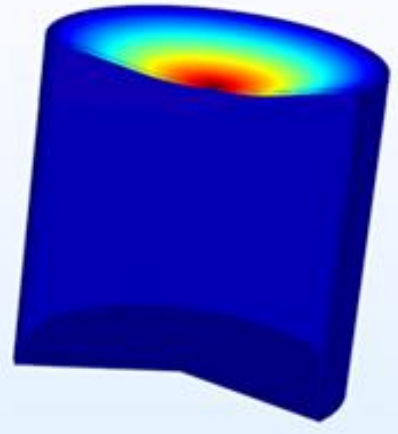

(b)

Figure 1 - (a) Conceptual drawing of waveguide-coupled microwave cylindrical cavity with flexible membrane. (b) Deflection of membrane due to dynamic fluid pressure changes cavity volume, which shifts microwave resonant frequency.

The work described in this report consists of preliminary investigation of transducer design parameters, which is performed through analytic derivations and computer simulations with COMSOL software package. This includes a study of signal sensitivity to displacement of the membrane due to dynamic fluid pressure, and dependence of sensitivity on transducer geometrical parameters. Coupling of microwave field into the cavity is briefly discussed as well. Once the size of cavity and optimal resonant mode are identified, a cavity design is proposed for fabricating a cavity prototype for proof-of-principle tests. 


\section{Sensor Model}

\subsection{Microwave resonant frequency shift}

The model of the sensor combines elements of fluid and solid mechanics, and electrodynamics. Dynamic pressure due to incompressible fluid flow normal to the membrane is given by Bernoulli equation:

$$
P=\frac{1}{2} \rho \mathrm{v}^{2}
$$

Where $\rho$ and $v$ are fluid density flow velocity, respectively. For uniform pressure, deflection of circular membrane of radius $R$ as a function of radial position $r$ can be calculated using Timoshenko's model for radially constrained circular plate [9]

$$
w(r)=\frac{P R^{4}}{64 D}\left(1-\frac{r^{2}}{R^{2}}\right)^{2}=w_{0}\left(1-\frac{r^{2}}{R^{2}}\right)^{2}
$$

Where $D$ is material fexural rigidity given as

$$
D=\frac{E d^{3}}{12\left(1-v^{2}\right)}
$$

Here $d$ is the plate thickness, $E$ and $v$ are the plate material Young's modulus and Poisson ratio, respectively. Maximum plate deflection occurs at the center

$$
w(0)=w_{0}=\frac{P R^{4}}{64 D}=\rho \mathrm{v}^{2} \frac{3\left(1-v^{2}\right)}{32 E} \frac{R^{4}}{d^{3}}
$$

and the average plate deflection is given as

$$
w_{\text {avg }}=\frac{1}{\pi R^{2}} \int_{0}^{2 \pi} \int_{0}^{R} w(r) r d r d \phi=\frac{w_{0}}{3}
$$

Resonant frequencies of transverse electric $T E_{n m l}$ modes of a cylindrical cavity are characterized by integers $n, m, l[7,8]$

$$
f_{n m l}^{T E}=\frac{c}{2 \pi \sqrt{\mu_{r} \varepsilon_{r}}} \sqrt{\left(\frac{X_{n m}^{\prime}}{R}\right)^{2}+\left(\frac{l \pi}{L}\right)^{2}}
$$

in which $\mu_{r}$ and $\varepsilon_{r}$ are permeability and permittivity of the medium, and $X_{n m}^{\prime}$ is the $n^{\text {th }}$ root of the derivative of the $m^{\text {th }}$ order Bessel function. The TM modes are described by a similar equation, with the difference that $X_{n m}^{\prime}$ is replaced with $X_{n m}$, the $n^{\text {th }}$ root of the $m^{\text {th }}$ order Bessel function. To prevent high-temperature fluid leaks into the sensor the cavity is pressurized with a buffer gas (e.g. Nitrogen or Helium). For gas-filled cavity, $\mu_{r}=1$ and $\varepsilon_{r}=1$. The quality factor $Q$ of a cavity is defined as 


$$
Q=\frac{f_{0}}{\Delta f_{F W H M}}
$$

where $f_{0}$ is the resonant frequency, and $\triangle f_{F W H M}$ is the full width at half maximum spectral linewidth of the cavity. The $Q$ of a gas-filled cavity is related to resisitve losses at the walls, which are determined by the material electrical conductivity. When optimizing $\mathrm{Q}$ with respect to cavity geometry, the maximum $\mathrm{Q}$ value for low order modes such as $\mathrm{TE}_{01 \mathrm{p}}$, is achieved when $L=2 R$. The cavity resonant frequency can be characterized by a single parameter

$$
f_{n m p}^{T E}=\frac{c}{2 \pi L} \sqrt{\left(2 X_{n m}^{\prime}\right)^{2}+(l \pi)^{2}}=\frac{c}{2 L} g^{T E}(n, m, l)
$$

and simimarly for TM mode frequencies, where $\mathrm{g}^{\mathrm{TE}}(\mathrm{n}, \mathrm{m}, \mathrm{l})$ and $\mathrm{g}^{\mathrm{TM}}(\mathrm{n}, \mathrm{m}, \mathrm{l})$ are non-dimensional function of the mode number integers n,m,l. Typical value of $Q$ for the low order mode of the cylindrical cavity is approximately 20,000 .

For small changes in the cavity hight due to membrane displacement of magnitude $\Delta L$, frequency shift is given, to first order in $\Delta L$, as

$$
\Delta f=\frac{d f}{d L} \Delta L=-\frac{c}{2 L^{2}} \Delta L g(n, m, l)
$$

For $\Delta L=-w_{\text {avg }}=-w_{0} / 3$, and using $L=2 R$,

$$
\Delta f=g(n, m, l) \frac{1-v^{2}}{256 E} \frac{c R^{2}}{d^{3}} \rho \mathrm{v}^{2}
$$

With respect to geometrical parmeters of the cavity, detection sensitivity increases quadratically with $R$, and inverse cubic with $d$. With respect to fluid parameters, detction sensitivity increases linearly with density and quadratically with velocity.

\subsection{Thermophysical material properties}

The properties of materials and fluids of variables which enter Equation (10) are functions of temperature. For stainless steel 316, Young's modulus $E$, shear modulus $G$, and Poisson ratio $v$ are given as [10]

$$
\begin{aligned}
& E=2.137 \times 10^{5}-102.74 T \\
& G=8.964 \times 10^{4}-53.78 T \\
& v=E / 2 G-1
\end{aligned}
$$

Where $E$ and $G$ are given in MPa, $T$ is given in ${ }^{\circ} \mathrm{C}$, and the correlations are valid for $0<T<800^{\circ} \mathrm{C}$.

Temperature-dependent values of liquid sodium density $\rho$ in units of $\mathrm{kg} / \mathrm{m}^{3}$ is given by the correlation [11]

$$
\rho=\rho_{c}+f\left(1-T / T_{c}\right)+g\left(1-T / T_{c}\right)^{h}
$$


Valid for $371 \mathrm{~K}<T<2503.7 \mathrm{~K}$, where $\rho_{c}=219, f=275.32, g=511.58, \mathrm{~h}=0.5, T_{c}=2503.7 \mathrm{~K}$.

For molten salt, density decreases linearly with temperature. For example, for FLiBe (LiF$\mathrm{BeF}_{2}$ ), density correlation in units of $\mathrm{kg} / \mathrm{m}^{3}$ is given by [12]

$$
\rho=2415.6-0.49072 T
$$

Where $T$ is in units of $\mathrm{K}$. The correlation is valid for $732.2 \mathrm{~K}<T<4498.8 \mathrm{~K}$. 


\section{Computer Simulations of Sensor Performance}

\subsection{Selection of transducer geometric parameters}

Thickness of the membrane is a critical parameter that affects sensor sensitivity. The value of thickness of the membrane is selected as a tradeoff between sensitivity and structural integrity of the sensor. One of the possible pathways for sensor failure is due to corrosion of the membrane. Prior studies indicate that stainless steel 316 is highly resilient to corrosion in liquid sodium. The data is usually based on converting the weight change of thin samples to loss rates in mils per year, and thus might not account for local inter-granular penetration damage which could affect the mechanical properties. The data indicates that corrosion of stainless steel in liquid sodium depends on the concentration of oxide impurities, sodium flow velocity, and temperature. One study showed that, when the maximum temperature of the liquid sodium is $600^{\circ} \mathrm{C}$, the oxide level is less than $10 \mathrm{ppm}$, and the velocity is $2 \mathrm{~m} / \mathrm{s}$, the corrosion rate at 2500 hours is approximately $2.3 \mu \mathrm{m} /$ year [13]. If the velocity is increased to $6 \mathrm{~m} / \mathrm{s}$, the corrosion rate is approximately $2.8 \mu \mathrm{m} /$ year, indicating that the velocity has only a slight effect on the corrosion rate. However, if the velocity is held at $6 \mathrm{~m} / \mathrm{s}$, and the maximum temperature is increased to $720^{\circ} \mathrm{C}$, the corrosion rate is increased roughly by one order of magnitude to approximately $30.2 \mu$ year. In the case of molten salt, $2 \mu \mathrm{m} /$ year corrosion rate of stainless steel 316 was observed in purified $\mathrm{FLiBe}\left(2 \mathrm{LiF}-\mathrm{BeF}_{2}\right)$ at $650^{\circ} \mathrm{C}$ in a static cell (no flow) $[14,15]$. However, another study of SS316 in FLiBe estimated the corrosion rate at $700^{\circ} \mathrm{C}$ estimated the corrosion rate to be between $17.1 \mu \mathrm{m} / \mathrm{year}$ and $31.2 \mu \mathrm{m} / \mathrm{year}$. In the liquid sodium environment of the Mechanisms Engineering Test Loop (METL) [16], the operating conditions are maximum temperature of $650^{\circ} \mathrm{C}$, liquid sodium velocity in pipes under $1 \mathrm{~m} / \mathrm{s}$, and oxide concentration of $<5 \mathrm{ppm}$. The corrosion rate of SS316 is expected to be approximately $0.1 \mathrm{mil} / \mathrm{year}$. Thus, in a 10year life span, the sensor membrane, on average, could corrode by $1 \mathrm{mil}$. We choose $d=10 \mathrm{mil}$ $(254 \mu \mathrm{m})$, so that corrosion would affect no more than $10 \%$ of the membrane. This number corresponds to a commonly available stainless steel stock sheet.

Another parameter that determines sensor sensitivity is the radius of the membrane. Besides the quadractic dependence on the radius in Equation (10), for a given mode, the size of the resonator determines the values of the resonant frequency. Fluid flow sensing is accomplished by measuring the shift of the resonant frequency caused by membrane displacement. The measurement procedure would involve scanning over the frequency span of $\triangle f_{F W H M}$ centered at $f_{0}$, with frequency resolution bandwidth $f_{R B W}$ of the spectrum analyzer readout. The trace sweep time $t_{\text {sweep }}$ would contribute to the flow meter time constant. Note that for a given value $f_{R B W}$ and $t_{\text {sweep }}$ are inversely related. For example, for of $L=2 R=0.8$ in $(2 \mathrm{~cm})$ gives $\mathrm{f}_{0} \sim 20 \mathrm{GHz}$. Using $\mathrm{Q}=20,000$, we obtain and $\Delta f_{\mathrm{FWHM}} \sim 1 \mathrm{MHz}$. For such parameters, a modern state-of-the-art power network analyzer (PNA) can perform the frequency sweep with $f_{\mathrm{RBW}}=3 \mathrm{KHz}$ and $t_{\text {sweep }}=62 \mathrm{~ms}$. The condition for dectability is $\Delta f>f_{\mathrm{RBW}}$. 


\subsection{Computer simulations of microwave resonant frequency shift}

To confirm validity of the analytical model approximations, frequency shift in a microwave cavity was calculated with COMSOL RF module. The modes of cylindrical cavity in order of increasing frequency are $\mathrm{TM}_{010}, \mathrm{TM}_{011}, \mathrm{TM}_{012}, \mathrm{TE}_{011}, \mathrm{TE}_{012}$, etc. The lowest frequency mode $\mathrm{TM}_{010}$ has no variation of the E-field with length, and therefore it is almost insensitive to length change. The subsequent modes, however, are sensitive to cavity volume change resulting from membrane displacement. COMSOL simulation results for a $R=0.4$ in and $d=10$ mil membrane cylindrical cavity in Figure 2 show spatial distribution of the electric fields in the cavity for $\mathrm{TM}_{011}$ mode, occurring at $15.4 \mathrm{GHz}$. The flexible membrane is at the top of the cylinder.

Using stainless-steel and liquid sodium material property values at $500^{\circ} \mathrm{C}$ given by Equations (11) and (12), respectively, we estimate $\Delta \mathrm{f}$ for a range of values velocity values from $0.5 \mathrm{~m} / \mathrm{s}$ to $2 \mathrm{~m} / \mathrm{s}$. Frequency shifts were calculated with COMSOL RF module for TM $\mathrm{M}_{011}$ mode. COMSOL simulations were performed for parabolic membrane deflection profile given by Equation (2), and for flat membrane displacement with amplitude $w_{\text {avg }}=w_{0} / 3$ according to Equation (5). For comparison, frequency shift was also calculated with the analytical model of Equation (10). The results are plotted in Figure 3. Notice that the COMSOL RF calculations for parabolic membrane deflection shape and for equivalent amplitude flat membrane displacement agree closely, and also agree reasonably well with frequency shifts calculated with the analytical model of Equation (10).

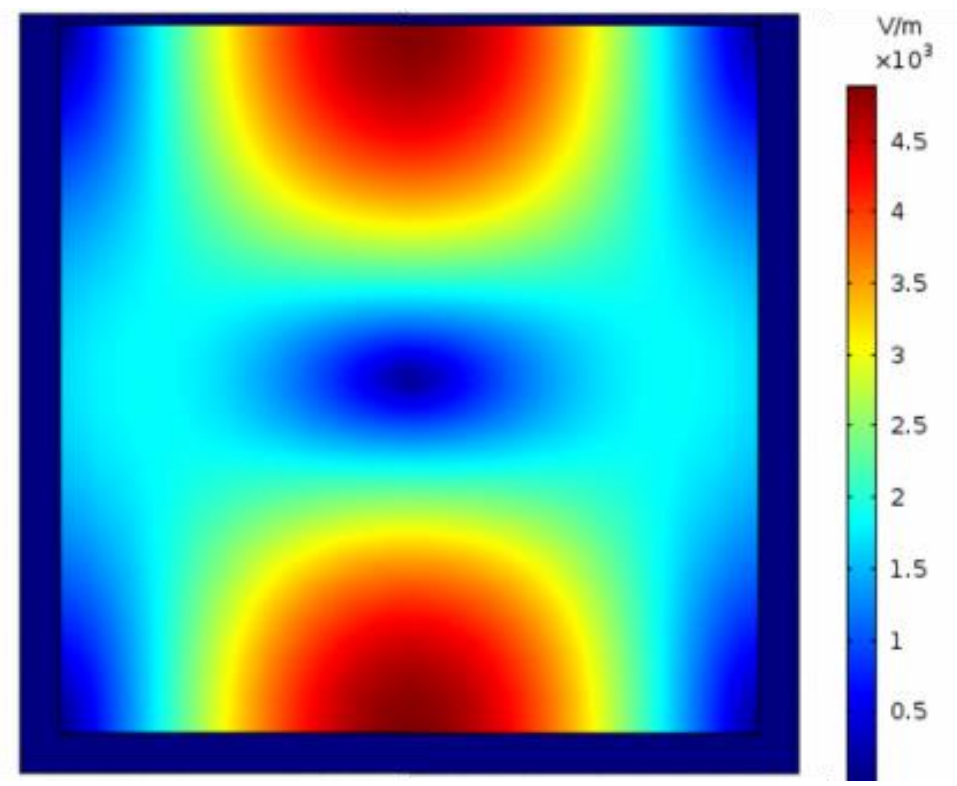

Figure 2 - Visualization of $\mathrm{TM}_{011}$ mode in cylindrical cavity with $R=0.8 \mathrm{in}$ occuring at $15.4 \mathrm{GHz}$. Flexible membrane with $d=10 \mathrm{mil}$ is at the top of the cylinder 


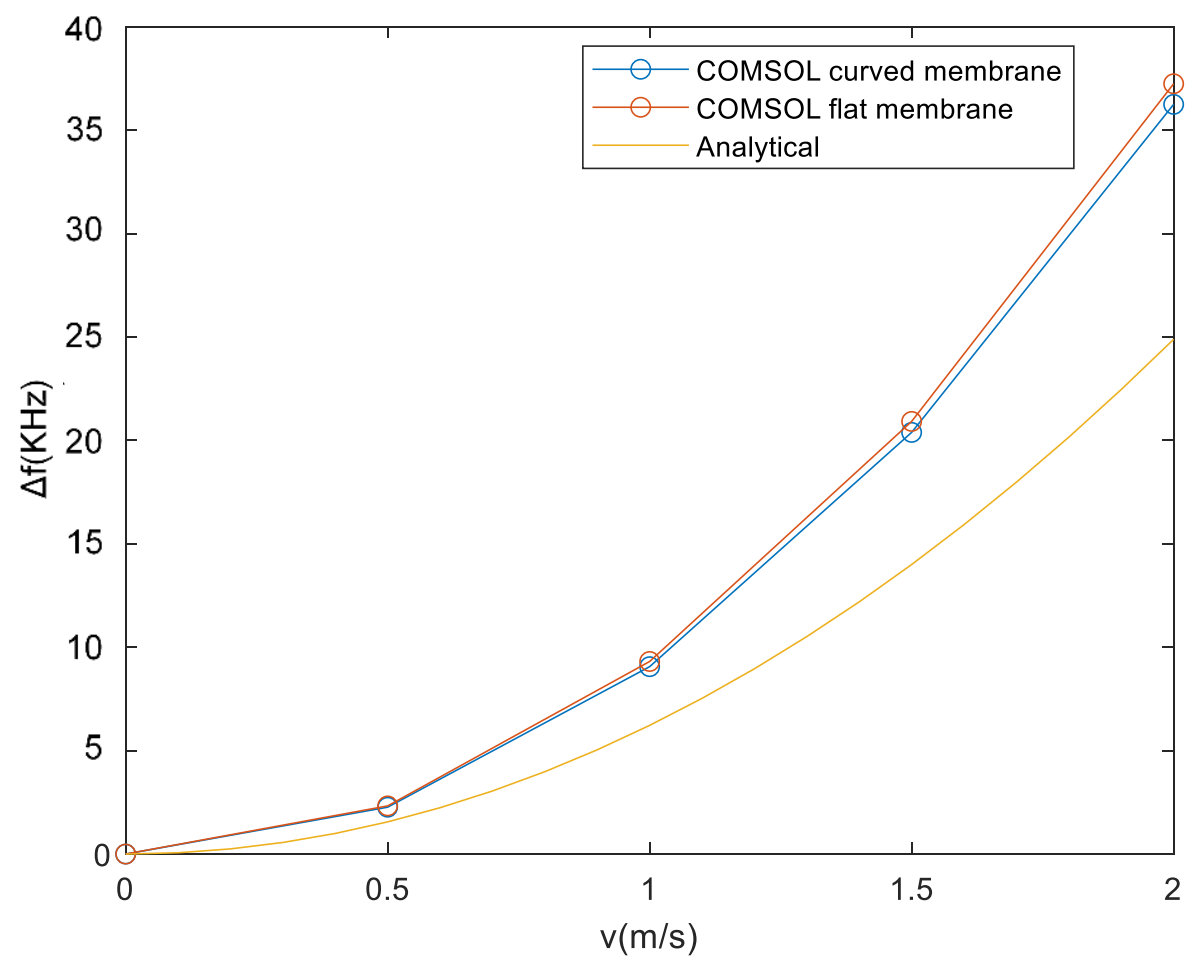

Figure 3 - (a) Conceptual Figure 3. Frequency shift of TM011 mode resonant frequency of $R=0.4$ in and $d=10$ mil stainelss steel cylinder due to liquid sodium flow-induced membrane deflections at $500^{\circ} \mathrm{C}$. Frequency shifts are calculated with COMSOL RF module using curved membrane model Equation (2), with COMSOL RF using flat membrane model with equivalent amplitude $w_{\text {avg }}=$ $w_{0} / 3$, and analytically using Equation (10).

Higher frequency modes, such as $\mathrm{TE}_{011}$ and $\mathrm{TE}_{012}$ modes, are known to have higher $\mathrm{Q}$ values than $\mathrm{TM}$ modes. Thus, we expect $\mathrm{TE}_{011}$ and $\mathrm{TE}_{012}$ modes to have better sensitivity to membrane displacement. Signal sensitivity for these two modes has been investigated through computer simulations, with results shown in Figures 4,5,6, and 7. COMSOL simulation results for $R=0.4$ in and $d=10 \mathrm{mil}$ right cylindrical cavity in Figure 4 show spatial distribution of the electric fields in $\mathrm{TE}_{011}$ mode, occurring at $22.2 \mathrm{GHz}$ resonant frequency with $\mathrm{Q}=17,900$. Frequency shifts of the stainless steel cavity were calculated with COMSOL RF module for $\mathrm{TE}_{011}$ mode for a range of velocities $(0.5 \mathrm{~m} / \mathrm{s}$ to $2 \mathrm{~m} / \mathrm{s})$ of liquid sodium flow at $500^{\circ} \mathrm{C}$. The membrane defection profile was modeled with Equation (2). Results of frequency shift computer simulations are shown in Figure 5. As expected, for the same fluid velocity, frequency shifts of $\mathrm{TE}_{011}$ mode are larger than those of the $\mathrm{TM}_{011}$ mode.

COMSOL simulation results for $R=0.4$ in and $d=10$ mil right cylindrical cavity in Figure 6 show spatial distribution of the electric fields in $\mathrm{TE}_{012}$ mode, occurring at $26.5 \mathrm{GHz}$ resonant frequency with $\mathrm{Q}=19,500$. Frequency shifts for $\mathrm{TE}_{012}$ mode for a range of velocities $(0.5 \mathrm{~m} / \mathrm{s}$ to $2 \mathrm{~m} / \mathrm{s})$ of liquid sodium flow at $500^{\circ} \mathrm{C}$ are displayed in Figure 7. Because of the higher $\mathrm{Q}$ value and spatial distribution profile, frequency shifts for $\mathrm{TE}_{012}$ are higher than those of $\mathrm{TE}_{011}$ mode. As discussed 
in Section 3.1, frequency shifts larger than $3 \mathrm{KHz}$ are, in principle, detectable with modern electronic devices.

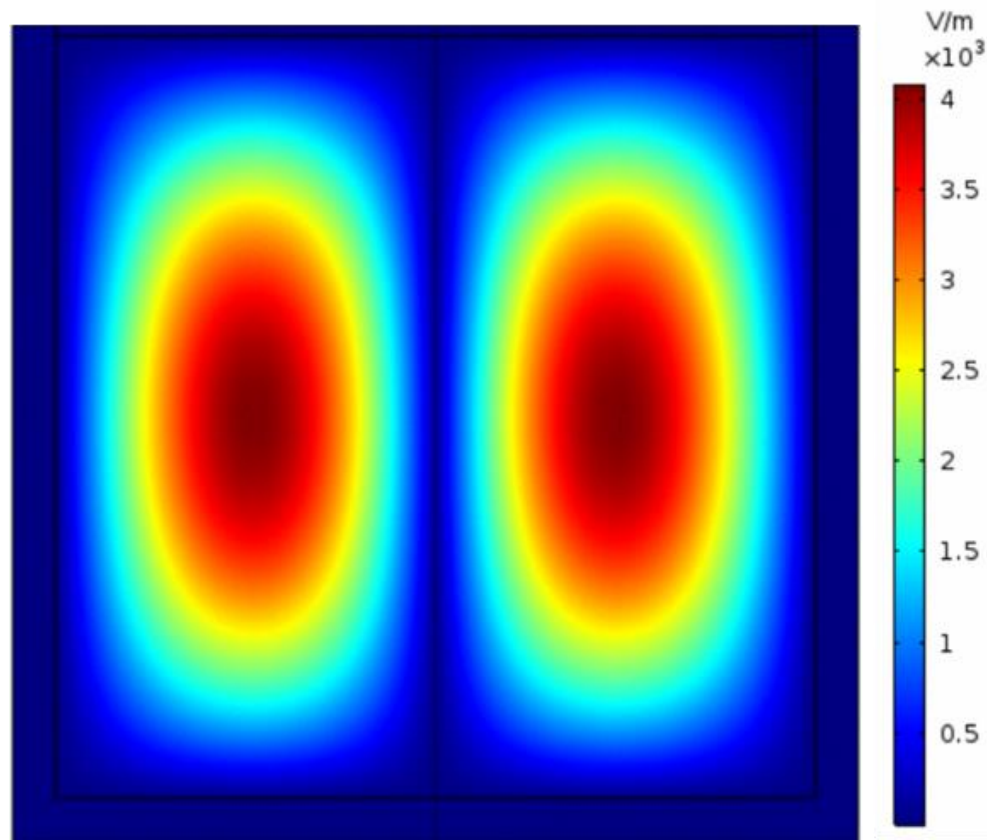

Figure 4 - Visualization of $\mathrm{TE}_{011}$ mode in right cylindrical cavity with $R=0.4$ in occuring at $f_{0}=$ $22.2 \mathrm{GHz}$. Flexible membrane with $d=10 \mathrm{mil}$ is at the top of the cylinder

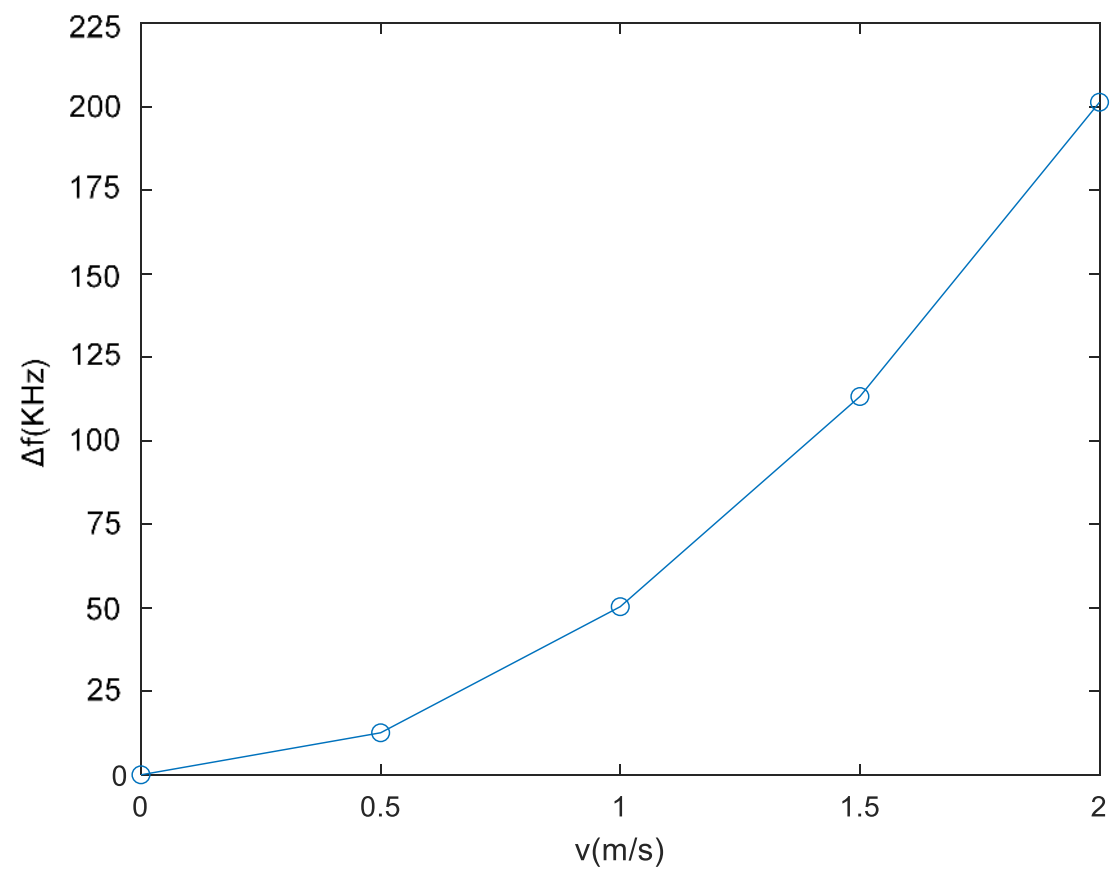

Figure 5 - Frequency shift of $\mathrm{TE}_{011}$ mode resonant frequency shift of $R=0.4$ in and $d=10 \mathrm{mil}$ stainless steel cylinder due to liquid sodium flow-induced membrane deflections at $500^{\circ} \mathrm{C}$. Frequency shift is calculated with COMSOL RF module using curved membrane model. 


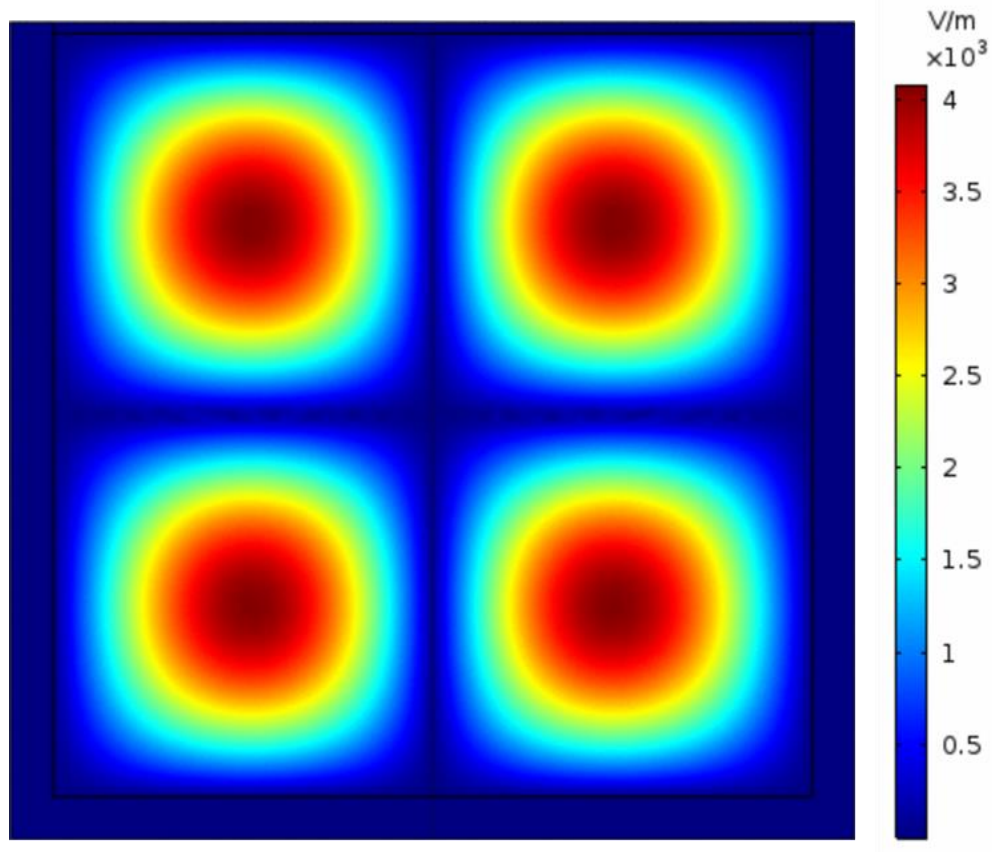

Figure 6 - Visualization of $\mathrm{TE}_{012}$ mode in right cylindrical cavity with $R=0.4$ in occuring at $f_{0}=$ $26.5 \mathrm{GHz}$. Flexible membrane with $d=10 \mathrm{mil}$ is at the top of the cylinder

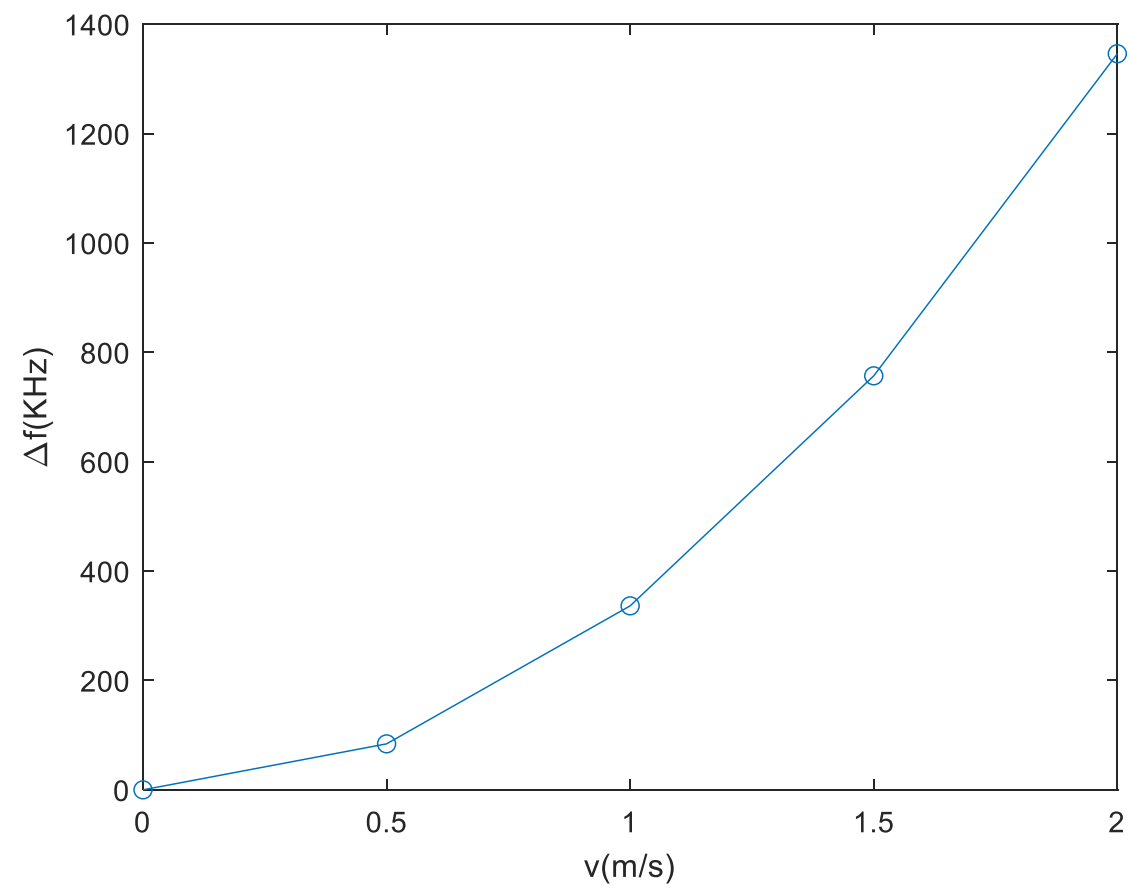

Figure 7 - Frequency shift of TE 012 mode resonant frequency shift of $R=0.4$ in and $d=10 \mathrm{mil}$ stainless steel cylinder due to liquid sodium flow-induced membrane deflections at $500^{\circ} \mathrm{C}$. Frequency shift is calculated with COMSOL RF module using curved membrane model 


\subsection{Coupling of microwave field into cylindrical resonator}

An important consideration for transducer design is the strategy for coupling microwave field into the cavity. As discussed in Section 1, our envisioned approach consists of coupling microwave field into the cylindrical resonator through a subwavelength-size aperture in the side wall of the cylinder. Selecting the size of an aperture involves a trade-off between coupling sufficient microwave energy into the cavity and degrading $Q$ factor of the cavity due to microwave leakage. Based on results of computer simulations in Section 2.2, best sensitivity to fluid velocity is expected for $\mathrm{TE}_{012}$ resonant mode. Considering spatial distribution of $\mathrm{TE}_{012}$, which is visualized in Figure 6, there is an electric field node in the mid-plane of the cavity. Thus, we anticipate that Q-factor of $\mathrm{TE}_{012}$ would display reasonable resilience to the size of the aperture. Quantitative dependence of $\mathrm{Q}$ factor on the aperture size for the $\mathrm{TE}_{012}$ is investigated with COMSOL RF module computer simulations, the results of which are shown in Figure 8. Note that the free space wavelength of the $\mathrm{TE}_{012}$ is $\lambda_{0}=1.13 \mathrm{~cm}$. One can observe in Figure 8 that the $\mathrm{Q}$ factor does not decrease significantly for $r<1.5 \mathrm{~mm}$, or $r<0.1 \lambda_{0}$. As $r$ increases above $1.5 \mathrm{~mm}$ value, $Q$ factor of the cavity is rapidly decreasing. This suggests the upper bound value on the coupling aperture value for the transducer design. Note that excitation of $\mathrm{TE}_{012}$ frequency in a cylindrical cavity can be accomplished with commercially available K-band $(18-26.5 \mathrm{GHz})$ metallic waveguides.

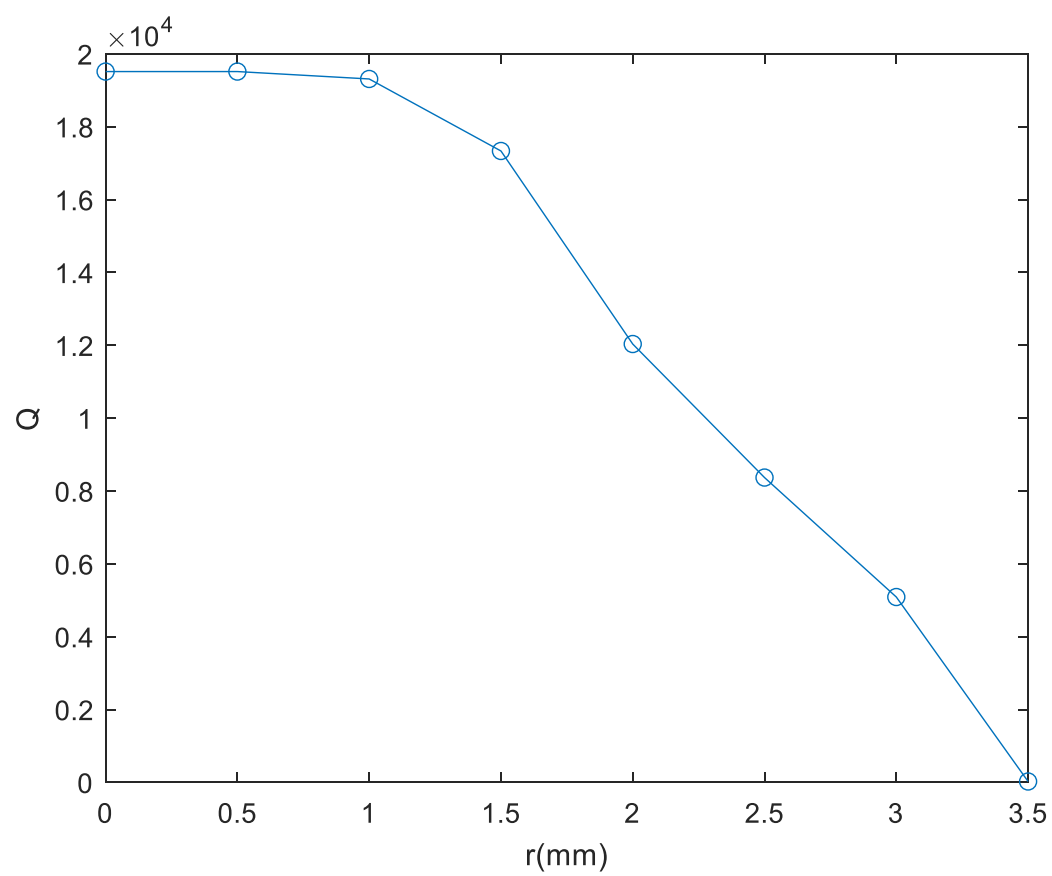

Figure 8 - Dependence of $\mathrm{Q}$ factor of $\mathrm{TE}_{012}$ mode on the radius of a circular aperture in the side wall of the cylindrical cavity 


\section{Preliminary Transducer Design}

Based on the results obtained from modeling and simulation of resonant transducer performance, preliminary design of the microwave cavity transducer was developed for fabrication in a machine shop. Because the resonant frequency of $\mathrm{TE}_{012}$ resonant mode discussed in Section 3 is in the K-band, the dimensions of the cylindrical right cavity were slightly adjusted to match those of the standard W/UG-595/U WR-42 waveguide flange (0.875in x 0.875 in square). Slight increase in cavity dimensions will result in slight decrease of the resonant frequency modes. The side view of the cavity is shown in Figure 9. All dimensions are in units of inches. In this design, the cavity is assembled with screws from a hollow cylinder, a thick cap at the bottom, and a thin diaphragm made from 10mil shim stock metal. The thin diaphragm is held in place with a clamping ring. All the holes in the drawing are for 4-40 screws, which are the same size screws used for Kband waveguide flange. In the design shown in Figure 6, microwave field is coupled into the cavity through a subwavelength aperture on the side of the cylinder. The diameter of the aperture is $3 \mathrm{~mm}$ (0.116in), which is the largest diameter that does not significantly degrade the $\mathrm{Q}$ factor of $\mathrm{TE}_{012}$ mode according to computer simulations in Section 3.3. The aperture is matched to the center of the waveguide. Coupling of waveguide to the cylindrical cavity is accomplished by chamfering the side of a cylindrical cavity to obtain a flat surface. 3D rendering of the cylindrical cavity in Figure 9 shows the chamfered side surface and the small aperture for coupling microwave field into cavity.

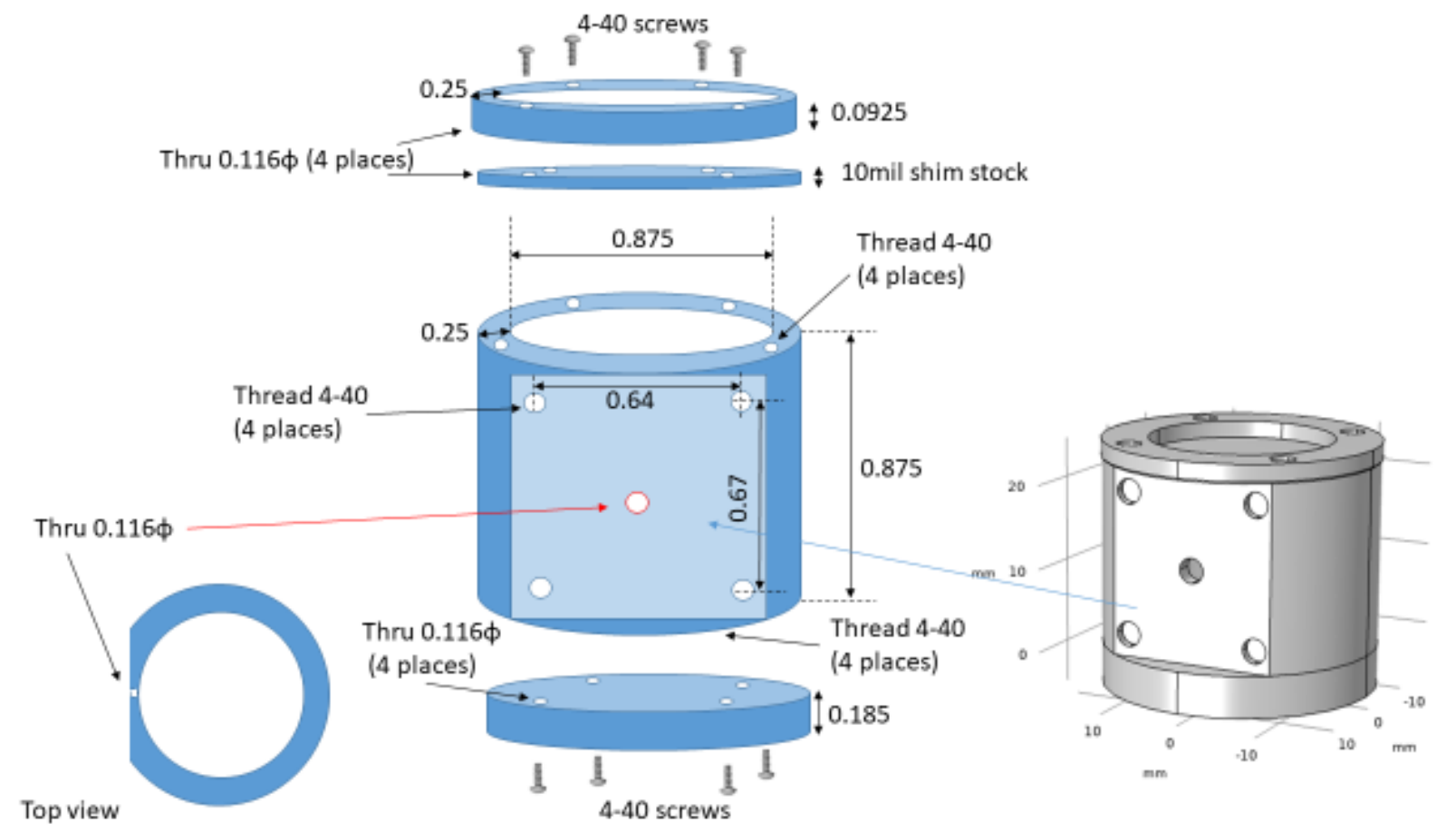

Figure 9 - Side view and 3D visualization of the cylindrical cavity design

Visualization of WR-42 microwave K-band waveguide attached to cylindrical cavity is shown in Figure 10. The top view of the cylinder without the diaphragm is also shown in Figure 10. In 
addition to the diameter of the aperture, another parameter influencing efficiency of microwave coupling into cavity is the thickness of the aperture screen. Selecting aperture thickness involves a tradeoff between coupling efficiency and mechanic structural integrity of the resonator. In the design shown in Figure 10, thickness of the aperture is 0.0925in (2.3mm). Dependence of coupling efficiency on screen thickness will be further investigated through computer simulations and proof-of-principle tests with coupons with apertures of different thicknesses.
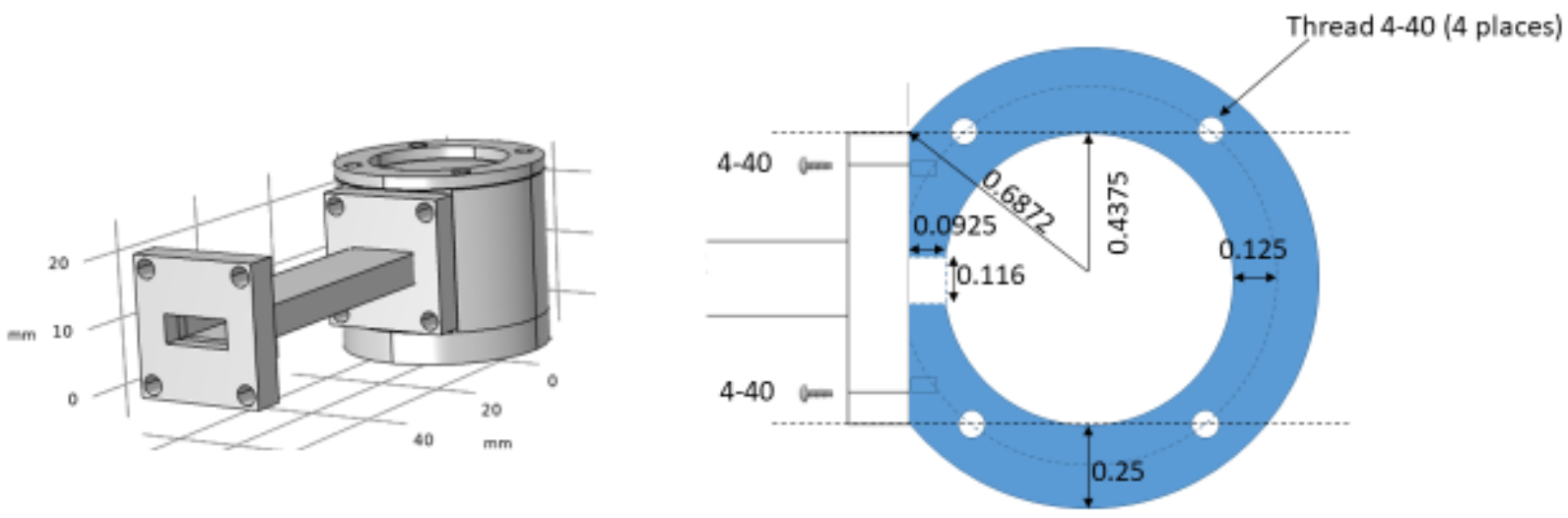

Figure 10 - Top view of the cavity and 3D visualization of waveguide coupling cavity side wall 


\section{Conclusions}

We have performed preliminary investigation of performance of a microwave cavity-based transducer for high-temperature fluid flow sensing using analytical derivations and computer simulations. This sensor is a hollow metallic cylindrical cavity, which can be fabricated from stainless steel, and as such is expected to be resilient to radiation, high temperature and corrosive environment of MSCR and SFR. The principle of sensing consists of making one wall of the cylindrical cavity flexible enough so that dynamic pressure, which is proportional to fluid velocity, will cause membrane deflection. A cavity is characterized by its resonant frequencies. Membrane deflection causes cavity volume change, which leads to a shift in the resonant frequency. Feasibility of the flow sensor is evaluated with signal sensitivity using COMSOL computer simulations. A right cylinder geometry stainless steel cavity with dimeter of $0.8 \mathrm{in}$ was investigated. We choose membrane thickness of $10 \mathrm{mil}$, so that corrosion anticipated to proceed at the rate of $1 \mathrm{mil} /$ year would affect no more than $10 \%$ of the membrane. Using the properties of liquid sodium fluid, and stainless-steel material property values at $500^{\circ} \mathrm{C}$, we calculate frequency shift for a range of values of fluid velocity from $0.5 \mathrm{~m} / \mathrm{s}$ to $2 \mathrm{~m} / \mathrm{s}$. Results of computer simulations indicate measurable sensitivity to flow for this transducer design. Computer simulations indicated the best sensitivity to flow-induced membrane displacement will be achieved when the cavity is excited in $\mathrm{TE}_{012}$ mode. Coupling of microwave filed into the cavity can be accomplished through a subwavelength size circular aperture on the cavity side wall. Computer simulations indicate that the $Q$ factor of the cavity is not affected significantly for cavity radius $r<0.1 \lambda_{0}$ (free space wavelength of $\mathrm{TE}_{012}$ mode. Following computer simulation results, transducer design was developed for fabrication of cavity prototype for proof-of-principle tests. For the design parameters identified in this study through computer simulation, the prototype cavity will operate in microwave K-band $(18-26.5 \mathrm{GHz})$. This cavity will be coupled with WR-42 K-band microwave waveguide. Future work will focus on sensor fabrication and tests to validate performance in a fluid. 


\section{References}

1. E. Blandford, K. Brumback, L. Fick, C. Gerardi, B. Haugh, E. Hillstrom, K. Johnson, P.F. Peterson, F. Rubio, F.S. Sarikurt, S. Sen, H. Zhao, N. Zweibaum, "Kairos Power Thermal Hydraulics Research and Development," Nuclear Engineering and Design 364, 110636 (2020).

2. C.W. Forsberg, P.F. Peterson, P.S. Pickard, "Molten-Salt-Cooled Advanced HighTemperature Reactor for Production of Hydrogen and Electricity," Nuclear Technology 144, 289-302 (2003).

3. K. Aoto, P. Dufour, Y. Hongyi, J. P. Glatz, Y. Kim, Y. Ashurko, R. Hill, N. Uto, “A Summary of Sodium-Cooled Fast Reactor Development," Progress in Nuclear Energy 77, 247-265 (2014).

4. K. Korsah, R.A. Kisner, C.L. Britton Jr., P. Ramuhalli, D.W. Woodan, N.C. Anheier, A.A. Diaz, E.H. Hirt, R.B. Vilim, H.T. Chien, S. Bakhtiari, S.H. Sheen, N. Gopalsami, A. Heifetz, S.K. Tam, Y. Park, B. Upadhyaya, A. Stanford, "Assessment of Sensor Technologies for Advanced Reactors,” PNNL-SA-132062 (2017).

5. A. Heifetz, D. Lisowski, M. Weathered, Y. Momozaki, H.T. Chien, S. Bakhtiari, "Preliminary Review Analysis of Distributed Sensors for Versatile Test Reactor (VTR) Environment," Argonne National Laboratory ANL/NSE-18/11 (2018).

6. D. Holcomb, R. Kisner, S. Cetiner, "Instrumentation Framework for Molten Salt Reactors," ORNL/TM-2018/868 (2018).

7. S. Bakhtiari, T. Gonnot, T. Elmer, H.T. Chien, D. Engel, E. Koehl, A. Heifetz, "Evaluation of Microwave Cavity Gas Sensor for In-Vessel Monitoring of Dry Cask Storage Systems," Proceeding of AIP Conference 1949, 110004 (2018).

8. A. Heifetz, V. Ankel, D. Shribak, S. Bakhtiari, A. Cilliers, "Microwave Resonant CavityBased Flow Sensor for Advanced Reactor High Temperature Fluids," $12^{\text {th }}$ Nuclear Plant Instrumentation, Control and Human-Machine Interface Technologies (NPIC-HMIT 2021) (2021).

9. S. Timoshenko and S. Woinowsky-Krieger, Theory of Plates and Shells, $2^{\text {nd }}$ Edition, McGraw- Hill, New York, (1964).

10. G.L. Hoffman, M.C. Billone, J.F. Koening, J.M. Kramer, J.D.B. Lambert, L. Leibowitz, Y. Orechwa, D.R. Pedersen, D.L. Porter, H. Tsai, and A.E. Wright, "Metallic Fuels Handbook," ANL-NSE-3 (2019).

11. J.K. Fink and L. Leibowitz, "Thermodynamic and Transport Properties of Sodium Liquid and Vapor," ANL/RE-95/2 (1995).

12. M.S. Sohal, M.A. Ebner, P. Sabharwall, P. Sharpe, "Engineering Database of Liquid Salt Thermophysical and Thermochemical Properties," INL/EXT-10-18297 (2010).

13. S.L. Schrock, J.N. Baysden, R.L. Miller, D.E Lohr, "Sodium Corrosion of Westinghouse Liquid Metal Fast Breeder Reactor (LMFBR) materials” In: Draley J.E., Weeks J.R. (eds) Corrosion by Liquid Metals. Springer, Boston, MA (1970). 
14. G. Zheng, B. Kelleher, G. Cao, M. Anderson, T. Allen, K. Sridharan, "Corrosion of 316 stainless steel in high temperature $\mathrm{Li}_{2} \mathrm{BeF}_{4}$ (FLiBe) salt," Journal of Nuclear Materials 461, 143-150 (2015).

15. E. Sooby-Woods, M. Alamaniotis, A. Heifetz, "Gaussian Process Ensemble for Corrosion Modeling and Prediction in Molten Salt Reactors," $12^{\text {th }}$ Nuclear Plant Instrumentation, Control and Human-Machine Interface Technologies (NPIC-HMIT 2021) (2021).

16. D. Kultgen, C. Grandy, E. Kent, M. Weathered, D. Andujar, and A. Reavis, "Mechanisms Engineering Test Loop - Phase I Status Report - FY2018," ANL-ART-148, ANL-METL-14 (2018). 


\section{Argonne}

Nuclear Science and Engineering (NSE) Division

Argonne National Laboratory

9700 South Cass Avenue, Bldg. 208

Argonne, IL 60439

www.anl.gov

Argonne National Laboratory is a U.S. Department of Energy laboratory managed by UChicago Argonne, LLC 